\title{
LOCAL INFECTION WITH PASTEURELLA SEPTICA AFTER A DOG BITE
}

\author{
BY \\ H. E. EMSON \\ From the Pathology Department, Birmingham Accident Hospital
}

(RECEIVED FOR PUBLICATION APRIL 27, 1956)

Pasteurella septica is a common pathogen of animals and birds, generally causing a haemorrhagic septicaemia, and it has been described as an upper respiratory tract commensal organism in cats, rats, and dogs. Human infection has been reported on several occasions following the bites of cats, the first recorded case being that of Kapel and Holm (1930), and occasionally from the bites of other animals, but only four cases following dog bites have been recorded (Allott, Cruickshank, Cyrlas-Williams, Glass, Meyer, Straker, and Tee, 1944 ; Brunsdon and Mallett, 1953). This is remarkable in view of the fact that the organism can be isolated from the tonsils of $50 \%$ of healthy dogs (Smith, 1955). A further case of local infection after a dog bite is reported here.

\section{Clinical History}

Mrs. E. R., aged 59, was bitten by a dog which had been frightened by fireworks on November 5, 1955. Seen 30 minutes after the accident, there were two ragged wounds on the dorsum of the right wrist, neither of which penetrated deeper than the subcutaneous fat. The wounds were trimmed and sutured under local anaesthesia, the arm fixed in a plaster slab and sling, and 1,500 units of anti-tetanus serum given after a test dose.

On November 16,1955, the centre of the wound was noted to be sodden; the sutures were removed, and the area cleaned with CTAB and redressed. By November 19 the centre of the wound had broken down over an area $3 \times 1 \mathrm{~cm}$., and the patient was admitted for excision and grafting. A swab was taken from the wound, and on culture it yielded a mixed growth of coagulase-positive Staphylococcus aureus and an organism subsequently identified as Pasteurella septica. At operation on November 21 the area was excised and a Thiersch graft from the forearm applied. On December 3 about $60 \%$ of the graft had taken, and the area was swabbed and redressed with penicillin cream. Culture of the swab grew Staph. aureus only. By December 10 healing was complete except for a small, raw donor area, and this too had healed by December 14. Flexion of the two middle fingers was incomplete and remedial exercises were started but improvement was slow. The area was radiographed on January 14, 1956, but no abnormal bony appearances were seen. Blood for serological investigation was taken on the same day.

\section{Bacteriology}

The organism isolated on November 19, 1955, was a small Gram-negative cocco-bacillus. It was suspected to be Pasteurella septica because of its morphology, its failure to grow on MacConkey medium, and the source of the infection. Its cultural and biochemical characteristics confirmed the suspicion, and identification was corroborated by demonstrating cross agglutination between an antiserum prepared against the organism and known strains of $P$. septica and vice versa.

The organism was basically cocco-bacillary in form, but pleomorphic when grown on solid media with some filamentous forms. It was Gramnegative, and with methylene blue showed bipolar staining. Growth occurred on unenriched nutrient agar, but was very slow. On blood agar good growth was present after 24 hours; the colonies were low, convex, grey, and translucent with an entire edge, and $1.0-1.5 \mathrm{~mm}$. in diameter. After three days' incubation they became opaque and whitish grey, with a raised central area and a low periphery. Horse blood was not haemolysed but became slightly browned and cleared after three days. Additional $\mathrm{CO}_{2}$ had no effect on the growth, and the organism also grew under anaerobic conditions, though more slowly. The organism did not grow on MacConkey medium incubated for seven days at $37^{\circ} \mathrm{C}$., but grew well in broth and after 48 hours gave a diffuse turbidity. After four days a deposit was present which resuspended incompletely on shaking, giving ropy strands and floccules, but there was no surface ring or pellicle. The organism was non-motile after 18 hours' incubation in fresh culture at $22^{\circ}$ and $37^{\circ} \mathrm{C}$. In gelatin at $22^{\circ}$ a stab culture gave 
a fine filiform growth without liquefaction. Growth in peptone water with or without added "sugars" was good after 24 hours.

\section{Biochemical Reactions}

These are presented in tabular form and are compared with two strains of $P$. septica from cat bites made available by the National Collection of Type Cultures (NCTC 8489 and 8771) and with the published reactions of certain other strains previously described by other workers. All these strains were similar in morphology and cultural characteristics to the one now reported (now referred to as strain ER) with the exception of that of Bartley and Hunter (1947). These workers describe growth on MacConkey medium, a finding not confirmed by any other worker.

It will be noted that strain ER differs from all others in that mannitol is not fermented. This sugar has been constantly attacked by all strains isolated from human infections, though Bergey (1948) only states that mannitol is "usually" fermented. Smith (personal communication) states that failure to ferment mannitol is characteristic of canine strains of $P$. septica, only $15 \%$ of such strains attacking this sugar. However, of the four strains previously isolated from dog bites, three did ferment mannitol and the ability of the fourth was not recorded. Strain ER also fails to ferment xylose, but fermentation of this sugar is a less constant characteristic. Sorbitol is not attacked by strain ER or by certain other strains, though Bergey states that this sugar is always attacked and uses fermentation of sorbitol as a characteristic to differentiate between $P$. septica and $P$. pestis. Production of $\mathrm{H}_{2} \mathrm{~S}$ and reduction of methylene blue by the strain ER were both very slow.

\section{Serology}

Blood was taken from Mrs. E. R. on January 14, 1956, 71 days after the injury and 35 days after healing was complete. The serum agglutinated strain ER to a titre of 1 in 32 , NCTC 8771 to a titre of 1 in 8 , and NCTC 8489 not at all. The serum from Mrs. E. R. did not agglutinate randomly chosen strains of Bact. coli and staphylococci, and the three strains of $P$. septica were not agglutinated by randomly chosen human sera. There was some difficulty in making smooth suspensions of all strains, which showed a tendency for some cultures to autoagglutinate in saline.

Two sera numbered 0704 and 4307, prepared against strains of $P$. septica obtained from the throats of healthy dogs, were kindly supplied by

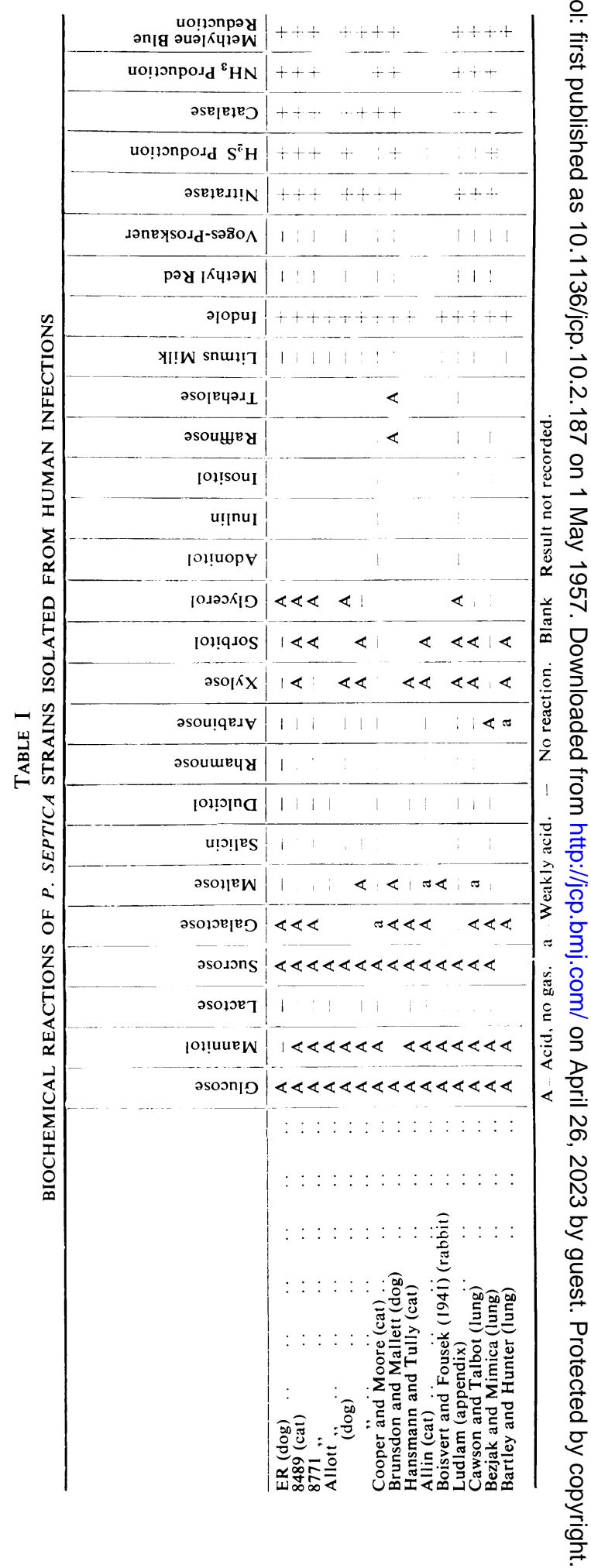


TABLE II

SEROLOGICAL REACTIONS OF CERTAIN STRAINS OF $P$. SEPTICA ISOLATED FROM HUMAN INFECTIONS

\begin{tabular}{|c|c|c|c|c|c|}
\hline \multirow[b]{2}{*}{ Organism } & & \multicolumn{4}{|c|}{ Antiserum } \\
\hline & & Mrs. E.R. & 0704 & 4307 & $\begin{array}{c}\text { Rabbit } \\
\text { Immunized } \\
\text { with ER }\end{array}$ \\
\hline $\begin{array}{l}\text { ER } \\
\text { NCTC } 8489\end{array}$ & . & $\begin{array}{r}132 \\
\mathrm{Nil}\end{array}$ & $\begin{array}{l}<130 \\
<+130\end{array}$ & $\begin{array}{l}<130 \\
+13 i l\end{array}$ & $\begin{array}{c}1 / 60 \\
1 / 120\end{array}$ \\
\hline , 8771 & . & 18 & $<130$ & ,, & $1 / 120$ \\
\hline
\end{tabular}

Mr. J. E. Smith of the Royal Veterinary College. Their reactions are set out in Table II. An antiserum to strain ER was prepared in a rabbit using a suspension of the organism in $0.5 \%$ phenolsaline as antigen. A course of four intravenous injections at weekly intervals raised the serum titre, originally 0 , to 1 in 60 against strain ER and 1 in 30 against NCTC 8771 and 8489. A further series of injections of the antigen did not succeed in raising the titre against strain $E R$, but that against NCTC 8771 and 8489 rose to 1 in 120 .

\section{Mouse Inoculation}

On November 24, 1955, two mice were inoculated with $0.2 \mathrm{ml}$. each of an overnight broth culture of strain ER. One mouse injected intraperitoneally was found dead next morning, and a necropsy showed pulmonary congestion. A Gramnegative cocco-bacillus identical with strain ER was recovered from peritoneal washings, heart blood, liver, spleen, and lung. The other mouse, injected intramuscularly, remained alive and well for 14 days. On November 28 two further mice were injected intraperitoneally, one with $0.1 \mathrm{ml}$. of a 1 in 10 dilution of an overnight broth culture, and one with $0.2 \mathrm{ml}$. of a 1 in 100 dilution. Both mice remained alive and well for 14 days.

\section{Discussion}

The literature on human infections with $P$. septica up to 1947 is summarized by Schipper (1947), who records among other cases 18 infections from cat bites, three from dog bites (those of Allott et al.), and one each from rabbit and panther bites. Since this date human infections in Great Britain have been recorded by Bartley and Hunter (1947), sinusitis ; Brunsdon and Mallett (1953), dog bite ; Pizey (1953), multiple arthritis in an infant; and Cawson and Talbot (1955), bronchiectasis. Bezjak and Mimica (1952) report two cases of upper respiratory tract infection from Jugoslavia, and refer to three other recent European reports.
Human infections were divided by Regamey (1939) into those following trauma, those following animal bites, and those of obscure origin. Bezjak and Mimica give figures of $50 \%, 10 \%$, and $40 \%$ for these three groups, and point out that of the last group many infections involve or are related to the upper respiratory tract. $P$. septica has been reported as an upper respiratory commensal in cats (Schenk, quoted by Allin, 1942; Hansmann and Tully, 1945), rats (Schipper, 1947), and dogs (Smith, 1955 ; carrier rate of $50 \%$ in healthy animals). Topley and Wilson (1955) mention an animal-house keeper who carried $P$. septica in his nose for several months without ill effect, and Needham is reported by Bezjak and Mimica as stating that he had isolated $P$. septica from 28 patients, nearly all with bronchiectasis. Several of the cases mentioned by Regamey are of trauma to the face or head followed by sinusitis or meningitis, and it seems not improbable that $P$. septica may occur as a human upper respiratory tract commensal. In two cases of sinusitis the organism was pathogenic and pyogenic, but in bronchiectasis its pathogenicity is in doubt, and Cawson and Talbot (1955) consider that it is probably only the colonizer of an already damaged bronchial tree.

Infections from animal bites have been characterized by prolonged course, slow healing, and a tendency to bone involvement. Antibodies to the organism are not commonly formed during such infections, and only Allott et al. (1944) record that in one case of dog bite infection the serum of the patient contained agglutinins for the infecting organism to a titre of 1 in 80 , and to a strain of $P$. septica from another dog bite infection to a titre of 1 in 20 . In systemic infection a high level of antibodies may be produced, as in Ludlam's (1944) case of appendix abscess.

\section{Classification}

When members of the group $P$. septica were first isolated from various animals they were named in terms of the infected animal; e.g., $P$. suiseptica, $P$. lepiseptica, etc. Further investigations emphasized the homogeneity of the group and the specific prefix was in general omitted, all being described as $\boldsymbol{P}$. septica ( $\boldsymbol{P}$. multocida). Subsequent attempts have been made to subdivide the group again, either on the basis of fermentative capabilities or on serological relationships.

Rosenbusch and Merchant (1939) excluded $P$. pestis and $P$. pseudotuberculosis and divided the other members of the Pasteurellae from animals as follows: 
Atypical strains, which haemolysed rabbit blood and fermented lactose, maltose, trehalose, raffinose, and inositol, were grouped as $P$. haemolytica. The remaining strains were grouped as $P$. septica and subdivided according to fermentation reactions:

Group I : Fermented arabinose and dulcitol, but not xylose.

Group II : Fermented xylose, but not arabinose or dulcitol.

Group III : Fermented all three sugars.

All fermented glucose, mannose, laevulose, and galactose, and none fermented rhamnose, salicin, inulin, dextrin or starch. Of the strains used to establish this classification, one was from a cat and one from a rabbit, but no dog strains were included.

Bezjak and Mimica quote Pestana and Rugai (1943) as stating that $97 \%$ of avian Pasteurella strains fermented arabinose, while mammalian strains failed to do so. They also quote Stamatin (personal communication) as stating that avian strains ferment arabinose but not xylose, while mammalian strains ferment xylose but not arabinose. Both strains described by these authors were of the avian type, but they proved nonpathogenic for pigeons and only one of their patients gave a history of contact with birds. Strains have been described from human infections which fermented neither of these sugars (ER, NCTC 8771, Cooper and Moore, 1945) while others fermented both (Bartley and Hunter, 1947). Of the strains recorded in Table I, not all the data are available, but by the classification of Rosenbusch and Merchant NCTC 8489, strains "Allin" and "Cawson and Talbot" fall into Group 2, and NCTC 8771, ER, "Ludlam" and "Cooper and Moore" are abnormal.

Serological subgrouping of $P$. septica was attempted by Cornelius (1929) working with 26 isolated animal strains, of which only one was from a cat and none of canine origin. He classified his strains into four groups; of the strains isolated from one species of animal, only those from mice fell together into the same group, and there was some suspicion that these strains were the same one isolated at different stages of an $\%$ experimental epidemic. Cornelius makes no $\overrightarrow{\vec{s}}$ reference to classification based on fermentative capabilities.

It appears that a classification of the $P$. septica group based on strains isolated primarily from $\overrightarrow{\widetilde{\sigma}}$ wild animals is inadequate to classify those isolated from human infections. The fermentative reactions of the group appear to be more diverse $\overrightarrow{0}$ than has been appreciated, and this, the variable $-\overrightarrow{-}$ agglutinability and the rapid loss of virulence $\omega_{\infty}$ described by some workers as occurring in laboratory cultures, may be related phenomena. They? may represent either distinct subdivisions of the $\vec{O}$ group, or possibly a high endogenous mutation? rate for these characteristics.

\section{Summary}

A case of localized human infection with Pasteureiia septica resulting from a dog bite is $\stackrel{0}{<}$ described.

The identification and classification of $P$. septica from human infections are discussed.

I am indebted to Sir William Gissane for permission to utilize the clinical notes of the patient undero his care, to Dr. S. Sevitt for help and encouragement, and to Mr. J. E. Smith, of the Royal Veterinary@ College, for unpublished data and the gift of sera.

\section{REFERENCES}

Allin, A. E. (1942). Canad. med. Ass. J., 46, 48.

Allott, E. N Cruickshank, R., Cyrlas-Williams, R., Glass, v: Meyer, I. H., Straker, E. A., and Tee, G. (1944). J. Path. Bact. 56, 411 .

Bartley, E. O., and Hunter, K. (1947). Lancet, 1, 908.

Bergey, D. H. (1948). Manual of Determinative Bacteriology, 6ttz ed. Baillière, Tindall and Cox, London.

Bezjak, V., and Mimica, M. (1952). Brit. med. J., 2, 757. Boisvert, P. L., and Fousek, M. D. (1941). J. Amer. med. Ass., 116

Brunsdon, D. F. V., and Mallett, B. L. (1953). Brit. med. J., 2, 607 Cawson, R. A., and Talbot, J. M. (1955). Journal of Clinical Patho$\log y, 8,49$

Cooper, T. V., and Moore, B. (1945). Lancet, 1, 753.

Cornelius, J. T. (1929). J. Path. Bact., 32, 355.

Hansmann, G. H., and Tully, M.(1945). Amer.J. clin. Path., 15, 3120

Kapel, O., and Holm, J. (1930). Zbl. Chir., 57, 2906.

Ludlam, G. B. (1944). J. Path. Bact., 56, 307.

Pestana, B. R., and Rugai, E. (1943). Rev. Inst. Adolfo Lutz, 3, 232

Pestana, B. R., (1953). Lancet, 2, 324.

Pegamey, R. (1939). Les Infections Humaines à B. bipolaris septicuß (Pasteurelloses). Huber, Berne. osenbusch, C. T., and Merchant, I. A. (1939). J. Bact., 37, 69. N

Schipper, G. J. (1947). Bull. Johns Hopk. Hosp., 81, 333.

Smith, J. E. (1955). J. comp. Path., 65, 239.

Topley, W. W. C., and Wilson, G. S. (1955). Principles of Bacteriology and Immunity, 4th ed. Arnold, London. 